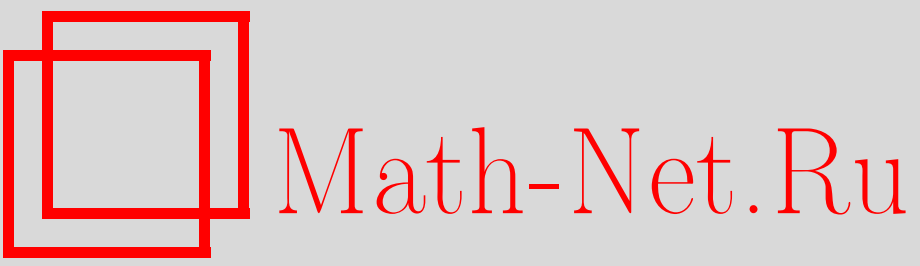

Т. В. Кузнецова, Уточнение предельной теоремы для максимумов независимых случайных сумм в случае нулевой асимметрии, Теория вероятн. и ее примен., 2010, том 55, выпуск 2, 357-362

DOI: https://doi.org/10.4213/tvp4206

Использование Общероссийского математического портала Math-Net.Ru подразумевает, что вы прочитали и согласны с пользовательским соглашением

http://www.mathnet.ru/rus/agreement

Параметры загрузки:

IP : 54.164 .48 .24

26 апреля 2023 г., 17:40:31 
4. Dupuis P., Ellis R.S. A Weak Convergence Approach to the Theory of Large Deviations. New York: Wiley, 1997, 479 p.

5. Freidlin M. I., Wentzell A.D. Random Perturbations of Dynamical Systems. New York: Springer-Verlag, 1984, $326 \mathrm{p}$.

6. Klebaner F. C. Introduction to Stochastic Calculus with Applications. London: Imperial College Press, 2005, 416 p.

7. Klebaner F. C., Liptser R. Likely path to extinction in simple branching models with large initial population. - J. Appl. Math. Stoch. Anal., 2006, Art. ID 60376, 23 p.

8. Liptser R. Sh., Pukhalskii A.A. Limit theorems on large deviations for semimartingales. - Stochastics Stochastics Rep., 1992, v. 38, № 4, p. 201-249.

9. Lu R., Hsu Y.-H. Valuation of standard options under the constant elasticity of variance model. - Internat. J. Business Econom., 2005, v. 4, № 2, p. 157-165.

10. Rogers L. C. G., Williams D. Diffusions, Markov Processes, and Martingales. V. 2. Itô Calculus. Cambridge: Cambridge Univ. Press, 2000, 480 p.

11. Rouault A. Large deviations and branching processes. - Pliska Stud. Math. Bulgar., 2000, v. 13 , p. $15-38$.

Поступила в редакцию 15.IV.2010

(c) 2010 г.

КУЗНЕЦОВА Т. В.*

\section{УТОЧНЕНИЕ ПРЕДЕЛЬНОЙ ТЕОРЕМЫ ДЛЯ МАКСИМУМОВ НЕЗАВИСИМЫХ СЛУЧАЙНЫХ СУММ В СЛУЧАЕ НУЛЕВОЙ АСИММЕТРИИ}

Рассматривается семейство экстремумов вида

$$
Y_{m n}=\max _{1 \leqslant i \leqslant m} \sum_{j=1}^{n} X_{i j}, \quad m, n \geqslant 1,
$$

где $\left\{X_{i j}\right\}, i \geqslant 1, j \geqslant 1$, - независимые одинаково распределенные случайные величины. Исследуется асимптотика $Y_{m n}$ при $m, n \rightarrow \infty$. Проводится уточнение предельной теоремы для случая, когда случайные величины $\left\{X_{i j}\right\}, i \geqslant 1, j \geqslant 1$, имеют нулевую асимметрию и, возможно, отличный от нуля эксцесс.

Ключевые слова и фразы: максимумы, случайные суммы, линейная нормировка, семиинвариант распределения порядка $k$, коэффициент эксцесса, коэффициент асимметрии.

1. Введение. В теории вероятностей и математической статистике широко используются следующие числовые характеристики распределений: коэффициент асимметрии (skewness) $S=\mu_{3} / \sigma^{3}$ и коэффициент эксцесса (kurtosis) $K=\mu_{4} / \sigma^{4}-3$, где $\mu_{k}=(\mathbf{E}(X-\mathbf{E} X))^{k}, \sigma^{2}=\mathbf{D} X$.

Для нормального распределения $S=0$ и $K=0$. Однако, как отмечено в [6, гл. 6], для реальных финансовых данных характерны большие положительные значения эксцесса (порядка единиц или десятков). Поэтому мы исследуем распределения, для которых $S=0$ и $K \neq 0$.

* Московский государственный университет им. М.В.Ломоносова, механикоматематический факультет, Ленинские горы, 119991 Москва, ГСП-1, Россия; e-mail: stv.msu@gmail.com 
В дальнейшем нам понадобится понятие семиинварианта. Рассмотрим функцию

$$
f(\zeta)=\int_{-\infty}^{\infty} e^{\zeta x} F\{d x\}
$$

где $F$ - функция распределения случайной величины $\zeta$. Предположим, что интеграл конечен при всех $\zeta$ из некоторого интервала $|\zeta|<\zeta_{0}$. Это равносильно тому, что характеристическая функция $\varphi(\zeta)=f(i \zeta)$ аналитична в некоторой окрестности нуля. Затем перейдем от функции $f$ к ее логарифму. Равенство

$$
\psi(\zeta)=\ln f(\zeta)=\sum \frac{\psi_{k}}{k !} \zeta^{k}
$$

определяет функцию, также аналитическую в некоторой окрестности нуля. Коэффициент $\psi_{k}$ зависит только от моментов распределения $F$ и носит название семиинварианта порядка $k$ распределения $F$. В общем случае $\psi_{1}=\mu_{1}, \psi_{2}=\sigma^{2}$.

Используем формулы для семиинвариантов, приведенные в [5, гл. II, $\S 12$, формулы (49), с. 402]:

$$
\psi_{3}=m_{3}-3 m_{1} m_{2}+2 m_{1}, \quad \psi_{4}=m_{4}-3 m_{2}^{2}-4 m_{1} m_{3}+12 m_{1}^{2} m_{2}-6 m_{1}^{4},
$$

где $m_{k}=\mathbf{E} \xi^{k}$. В случае $m_{1}=0$ получаем:

$$
\psi_{3}=m_{3}, \quad \psi_{4}=m_{4}-3 m_{2}^{2},
$$

или (с учетом того, что при $m_{1}=0$ верно $\left.m_{k}=\mu_{k}=\mathbf{E}(\xi-\mathbf{E} M \xi)^{k}\right)$

$$
\psi_{3}=\mu_{3}, \quad \psi_{4}=\mu_{4}-3 \sigma^{4} .
$$

При $m_{1}=0$ и $\sigma^{2}=1$ наблюдается равенство: $\psi_{3}=S, \psi_{4}=K$.

Рассмотрим семейство экстремумов вида

$$
Y_{m n}=\max _{1 \leqslant i \leqslant m} \sum_{j=1}^{n} X_{i j}, \quad m, n \geqslant 1,
$$

где $\left\{X_{i j}\right\}, i \geqslant 1, j \geqslant 1$, - независимые одинаково распределенные случайные величины с функцией распределения $F$. Нас интересует асимптотика $Y_{m n}$ при $m, n \rightarrow \infty$.

Основные результаты работы [1] (приводимые далее теоремы 1-3) получены при условии, что $F$ обладает конечным средним и дисперсией (которые для простоты полагаются равными нулю и единице соответственно).

Теорема 1. Если существуют $m_{3}, \ldots, m_{r}, r \geqslant 3, u m=O\left(n^{r / 2-1}\right), m, n \rightarrow \infty$, то существуют константы $a_{m} u b_{m}$ такие, что

$$
\mathbf{P}\left\{a_{m}\left(n^{-1 / 2} Y_{m n}-b_{m}\right) \leqslant x\right\} \rightarrow \exp \left\{-e^{-x}\right\} .
$$

Если же предположить аналитичность функции $f(z)$ в некоторой окрестности нуля, то это предположение существенно ослабит условия, налагаемые на рост $m$ по сравнению с $n$.

Теорема 2. Если $\ln m=o\left(n^{1 / 3}\right), m, n \rightarrow \infty$, то утверждение (2) верно.

Теорема 3. Если $\ln m=o(n), m, n \rightarrow \infty$, то существуют $\alpha_{m n} \sim \beta_{m n} \sim$ $(2 \ln m)^{1 / 2}$ maкue, umo

$$
\mathbf{P}\left\{\alpha_{m}\left(n^{-1 / 2} Y_{m n}-\beta_{m}\right) \leqslant x\right\} \rightarrow \exp \left\{-e^{-x}\right\} .
$$

Суммы в (1) оказываются асимптотически нормальными, а для максимумов независимых нормальных случайных величин имеет место предельный закон Гумбеля. Таким образом, при $\mathbf{E} X_{i j}=0, \mathbf{D} X_{i j}=1$ и некоторых ограничениях на относительный рост $m, n \rightarrow \infty$ имеет место сходимость

$$
\mathbf{P}\left\{\alpha_{m}\left(n^{-1 / 2} Y_{m n}-\beta_{m}\right) \leqslant x\right\} \rightarrow \exp \left\{-e^{-x}\right\},
$$


где $\alpha_{m}=(2 \ln m)^{1 / 2}, \beta_{m}=(2 \ln m)^{1 / 2}-(2 \ln m)^{-1 / 2} \ln (4 \pi \ln m)^{1 / 2}$.

Далее рассмотрим $Y_{m n}=\max _{1 \leqslant i \leqslant m} \sum_{j=1}^{n} X_{i j}, m, n \geqslant 1$, для случая, когда $\left\{X_{i j}\right\}$, $i \geqslant 1, j \geqslant 1$, - независимые одинаково распределенные случайные величины с функцией распределения $F$, имеющие нулевую асимметрию и, возможно, отличный от нуля эксцесс. Будем также предполагать, что $\mathbf{E} X_{i j}=0, \mathbf{D} X_{i j}=1$, а характеристическая функция $f$ аналитична в окрестности нуля. Для этого случая получим более точное выражение для констант $\alpha_{m n} \sim \beta_{m n} \sim(2 \ln m)^{1 / 2}$ таких, что $\mathbf{P}\left\{\alpha_{m}\left(n^{-1 / 2} Y_{m n}-\beta_{m}\right) \leqslant x\right\} \rightarrow \exp \left\{-e^{-x}\right\}$.

\section{2. Основной результат.}

Теорема 4. Пусть $Y_{m n}=\max _{1 \leqslant i \leqslant m} \sum_{j=1}^{n} X_{i j}, m, n \geqslant 1, X_{i j}$ - независимье случайнье величинь с распределением $F, \ln m=o\left(n^{6 / 10}\right), m, n \rightarrow \infty$. Тогда существуют константы $\alpha_{m n} \sim \beta_{m n} \sim(2 \ln m)^{1 / 2}$ такие, что $\mathbf{P}\left\{\alpha_{m}\left(n^{-1 / 2} Y_{m n}-\beta_{m}\right) \leqslant\right.$ $x\} \rightarrow \exp \left\{-e^{-x}\right\}$, и они выражаются следующими формулами:

$$
\beta_{m n}=\sqrt{2 \ln m}-\frac{\psi_{4}}{12 n} \ln m \cdot \sqrt{2 \ln m}-\frac{\ln 4 \pi+\ln \ln m}{2 \sqrt{2 \ln m}}, \quad \alpha_{m n}=\sqrt{2 \ln m} .
$$

Д о к а з а т е л ь с т в о. Существование констант следует из теоремы 3.

Обозначим $F_{n}(x)=\mathbf{P}\left\{S_{i n} n^{-1 / 2} \leqslant x\right\}$, где $S_{i n}=\sum_{j=1}^{n} X_{i j}$. c. 619]:

Если $u=o\left(n^{1 / 2}\right), m, n \rightarrow \infty$, то верно соотношение [2, гл. XVI, $\S 7$, теорема 2,

$$
\frac{1-F_{n}(u)}{1-\Phi(u)}=\exp \left\{u^{2} \lambda\left(\frac{u}{\sqrt{n}}\right)\right\}\left(1+O\left(\frac{u}{\sqrt{n}}\right)\right),
$$

где $\lambda(z)$ - функция, аналитическая в окрестности нуля, $\lambda(0)=0$. С учетом асимптотики хвоста нормального распределения получаем:

$$
1-F_{n}(u) \sim \frac{1}{\sqrt{2 \pi} u} \exp \left\{-\frac{u^{2}}{2} \gamma\left(\frac{u}{\sqrt{n}}\right)\right\}
$$

где функция $\gamma(z)=1-2 \lambda(z)$ аналитична в некоторой $\varepsilon$-окрестности нуля, $\gamma(0)=1$. Обозначим правую часть (3) через $h(u)$. Имеем $h(u) \rightarrow+\infty$ при $u \rightarrow 0$ и

$$
h\left(\varepsilon n^{1 / 2}\right)=\frac{1}{\varepsilon \sqrt{2 \pi n}} \exp \left\{-\frac{\varepsilon^{2}}{2} \gamma(\varepsilon) n\right\}=o\left(\frac{1}{m}\right), \quad m, n \rightarrow \infty .
$$

Поскольку $h(u)$ непрерывна на $\left(0, \varepsilon n^{1 / 2}\right)$, то для любого числа $x$ при достаточно больших $m, n$ существуют $u_{m n} \in\left(0, \varepsilon n^{1 / 2}\right)$ такие, что $h\left(u_{m n}\right)=e^{-x} / m$. $o\left(n^{3 / 10}\right)$.

По условию $\alpha_{m n} \sim \beta_{m n} \sim(2 \ln m)^{1 / 2}$, значит, $u_{m n}=\beta_{m n}+x / \alpha_{m n} \sim(2 \ln m)^{1 / 2} \sim$

Воспользуемся утверждением (см. [2, гл. XVI, §7, формула (7.29), с. 619]) о том, что если $u$ возрастает как $o\left(n^{3 / 10}\right)$, то достаточно ограничиться членами разложения функции $\lambda(z)$ в степенной ряд до второй степени:

$$
\lambda(z)=\lambda_{1} z+\lambda_{2} z^{2} .
$$

В рассматриваемом случае $\lambda_{1}=\mu_{3} /\left(6 \sigma^{3}\right)=0, \lambda_{2}=\left(\sigma^{2} \psi_{4}-3 \psi_{3}^{2}\right) /\left(24 \sigma^{6}\right)=\psi_{4} / 24$.

Таким образом, найдены функции $\lambda(z)$ и $\gamma(z)$ :

$$
\lambda(z)=\frac{\psi_{4}}{24} z^{2}, \quad \gamma(z)=1-\frac{\psi_{4}}{12} z^{2} .
$$

Подставив последнее выражение для $\gamma(z)$ в (3), получим уравнение:

$$
h(u)=\frac{1}{\sqrt{2 \pi} u} \exp \left\{-\frac{u^{2}}{2}+\frac{\psi_{4} u^{4}}{24 n}\right\} \sim \frac{e^{-x}}{m}, \quad m, n \rightarrow \infty .
$$


Прологарифмируем это выражение и получим:

$$
-\ln \sqrt{2 \pi}-\ln u-\frac{u^{2}}{2}+\frac{\psi_{4} u^{4}}{24 n}=-x-\ln m+o(1), \quad m, n \rightarrow \infty .
$$

Поскольку $u^{2} / 2=o\left(n^{6 / 10}\right)$, а $\psi_{4} u^{4} /(24 n)=o\left(n^{2 / 10}\right)$, то в левой части уравнения (4) главный член есть $u^{2} / 2$. Кроме того, так как $\ln m=o\left(n^{6 / 10}\right)$, то справедливо соотношение

$$
\frac{u^{2}}{2} \sim \ln m, \quad m \rightarrow \infty .
$$

Прологарифмировав его, получим выражение для $\ln u$ :

$$
2 \ln u-\ln 2=\ln \ln m+o(1), \quad \ln u=\frac{\ln \ln m+\ln 2}{2}+o(1) .
$$

Подставим это выражение в (4) и решим (4) как квадратное уравнение относительно $u^{2}$ :

$$
\begin{gathered}
-\ln \sqrt{2 \pi}-\frac{\ln \ln m+\ln 2}{2}-\frac{u^{2}}{2}+\frac{\psi_{4} u^{4}}{24 n}=-x-\ln m, \\
\frac{\psi_{4} u^{4}}{24 n}-\frac{u^{2}}{2}-\ln \sqrt{2 \pi}-\frac{\ln \ln m+\ln 2}{2}+x+\ln m=0, \\
\frac{\psi_{4}}{12 n} u^{4}-u^{2}-\ln \sqrt{2 \pi}-\ln \ln m+2 x+2 \ln m=0, \\
D=1+\frac{\psi_{4}}{3 n}(\ln 4 \pi+\ln \ln m-2 x-2 \ln m) .
\end{gathered}
$$

Учитывая порядки роста $\ln m$ и $\ln \ln m$, получаем, что

$$
\frac{\psi_{4}}{3 n}(\ln 4 \pi+\ln \ln m-2 x-2 \ln m)=o\left(n^{-4 / 10}\right), \quad n, m \rightarrow \infty .
$$

Поэтому, воспользовавшись формулой $\sqrt{1+x}=1+x / 2-x^{2} / 8+O\left(x^{3}\right), x \rightarrow 0$, корень из дискриминанта можно разложить в ряд Тейлора с точностью до первых трех членов:

$$
\begin{aligned}
\sqrt{D}= & 1+\frac{\psi_{4}}{6 n}(\ln (4 \pi)+\ln \ln m-2 x-2 \ln m) \\
& -\frac{\psi_{4}^{2}}{72 n^{2}}(\ln (4 \pi)+\ln \ln m-2 x-2 \ln m)^{2} \\
& +o\left(n^{-12 / 10}\right), \quad n, m \rightarrow \infty .
\end{aligned}
$$

Итак, рассмотрим корни уравнения $(4): u_{1,2}^{2}=\left((1 \pm \sqrt{D}) / \psi_{4}\right) \cdot 6 n$. Первый корень $u_{1}^{2}=\left((1+\sqrt{D}) / \psi_{4}\right) 6 n$ не подходит, так как $u^{2}=o\left(n^{6 / 10}\right)$, и в то же время, учитывая разложение $(5)$, получаем, что $u_{1}^{2} \sim\left(6 / \psi_{4}\right) n$. Таким образом, рассматриваем только второй корень:

$$
u_{2}^{2}=\frac{1-\sqrt{D}}{\psi_{4}} 6 n
$$

Подставим полученное в $(5)$ выражение для $\sqrt{D}$ :

$u^{2}=(2 x+2 \ln m-\ln (4 \pi)-\ln \ln m)-\frac{\psi_{4}}{12 n}(2 x+2 \ln m-\ln (4 \pi)-\ln \ln m)^{2}+o\left(n^{-2 / 10}\right)$, и раскроем скобки:

$$
\begin{aligned}
u^{2}= & (2 x+2 \ln m-\ln (4 \pi)-\ln \ln m) \\
-\frac{\psi_{4}}{12 n} & \left(4 x^{2}+4 \ln ^{2} m+8 x \ln m+(\ln \ln m)^{2}+\ln ^{2}(4 \pi)+2 \ln (4 \pi) \ln \ln m\right. \\
& \quad-4 x \ln \ln m-4 x \ln (4 \pi)-4 \ln m \ln \ln m-4 \ln m \ln (4 \pi))+o\left(n^{-2 / 10}\right) .
\end{aligned}
$$


Рассмотрим следующие слагаемые в правой части равенства:

$$
\begin{aligned}
& \frac{\psi_{4}}{12 n} \cdot 4 x^{2} \sim o\left(n^{-1}\right), \quad \frac{\psi_{4}}{12 n} \cdot 4 \ln ^{2} m \sim o\left(n^{2 / 10}\right), \quad \frac{\psi_{4}}{12 n} \cdot 8 x \ln m \sim o\left(n^{-4 / 10}\right), \\
& \frac{\psi_{4}}{12 n}(\ln \ln m)^{2} \sim \frac{O\left(\ln ^{2} n\right)}{n}, \quad \frac{\psi_{4}}{12 n} \ln ^{2}(4 \pi) \sim o\left(n^{-1}\right), \\
& \frac{\psi_{4}}{12 n} 2 \ln (4 \pi) \ln \ln m \sim \frac{O(\ln n)}{n}, \quad \frac{\psi_{4}}{12 n} \cdot 4 x \ln \ln m \sim \frac{O(\ln n)}{n}, \\
& \frac{\psi_{4}}{12 n} \cdot 4 x \ln (4 \pi) \sim o\left(n^{-1}\right), \quad \frac{\psi_{4}}{12 n} 4 \ln m \ln \ln m \sim O(\ln n) o\left(n^{-4 / 10}\right), \\
& \frac{\psi_{4}}{12 n} \cdot 4 \ln m \ln (4 \pi) \sim o\left(n^{-1}\right) .
\end{aligned}
$$

Как видим, всеми слагаемыми, кроме $\psi_{4}(12 n)^{-1} 4 \ln ^{2} m$, можно пренебречь, так как они стремятся к нулю при $m, n \rightarrow \infty$.

Таким образом, получим следующее выражение для $u^{2}$ :

$$
\begin{aligned}
u^{2} & =2 x+2 \ln m-\ln (4 \pi)-\ln \ln m-\frac{\psi_{4}}{3 n} \ln ^{2} m+o\left(n^{-2 / 10}\right) \\
& =2 \ln m \cdot\left(1+\frac{2 x-\ln (4 \pi)-\ln \ln m-\left(\psi_{4} /(3 n)\right) \ln ^{2} m}{2 \ln m}\right)+o\left(n^{-2 / 10}\right),
\end{aligned}
$$

и, значит,

$$
u=\sqrt{2 \ln m} \sqrt{\left(1+\frac{2 x-\ln (4 \pi)-\ln \ln m-\left(\psi_{4} /(3 n)\right) \ln ^{2} m}{2 \ln m}\right)+\frac{o\left(n^{-2 / 10}\right)}{2 \ln m}} .
$$

Заметим, что

$$
\frac{2 x-\ln (4 \pi)-\ln \ln m-\left(\psi_{4} /(3 n)\right) \ln ^{2} m}{2 \ln m}=o(1), \quad n, m \rightarrow \infty .
$$

Поэтому второй корень в (6) можно разложить в ряд Тейлора (используем точность разложения до первых двух членов, $\left.\sqrt{1+x}=1+x / 2+o\left(x^{2}\right), x \rightarrow 0\right)$ :

$$
u=\sqrt{2 \ln m}\left(1+\frac{2 x-\ln (4 \pi)-\ln \ln m-\left(\psi_{4} /(3 n)\right) \ln ^{2} m}{4 \ln m}+\frac{o\left(n^{-2 / 10}\right)}{2 \ln m}\right)
$$

при $m, n \rightarrow \infty$. Следовательно,

$$
u=\frac{x+o(1)}{\sqrt{2 \ln m}}-\frac{\ln (4 \pi)+\ln \ln m}{2 \sqrt{2 \ln m}}+\sqrt{2 \ln m}\left(1-\frac{\psi_{4}}{12 n} \ln m\right) .
$$

Вспоминая, что $u=u_{m n}=\beta_{m n}+(x+o(1)) / \alpha_{m n}$, находим выражения для констант $\beta_{m n}$ и $\alpha_{m n}$ :

$$
\beta_{m n}=\sqrt{2 \ln m}-\frac{\psi_{4}}{12 n} \ln m \cdot \sqrt{2 \ln m}-\frac{\ln (4 \pi)+\ln \ln m}{2 \sqrt{2 \ln m}}, \quad \alpha_{m n}=\sqrt{2 \ln m} .
$$

Теорема доказана.

Автор выражает признательность своему научному руководителю доценту А. В. Лебедеву за поставленные задачи и полезные замечания.

\section{СПИСОК ЛИТЕРАТУРЫ}

1. Лебедев А.В. Предельные теоремы для максимумов независимых случайных сумм. - Теория вероятн. и ее примен., 1999, т. 44, в. 3, с. 631-634. 
2. Феллер В. Введение в теорию вероятностей и ее приложения. Т. 2. М.: Мир, 1984. $752 \mathrm{c}$.

3. Лидбеттер М., Линдгрен Г., Ротсен Х. Экстремумы случайных последовательностей и процессов. М.: Мир, 1989, 391 с.

4. Галамбош Я. Асимптотическая теория экстремальных порядковых статистик. М.: Наука, 1984, 303 с.

5. Ширяев А.Н. Вероятность-1. М.: МЦНМО, 2007, 551 с.

6. Ширяев А.Н. Основы стохастической финансовой математики. Т. 1. М.: Фазис, $1998,489 \mathrm{c}$

Поступила в редакцию 21.X.2009

(c) 2010 г.

МУРАВЛЁВ А. А.*

\section{ОБ ОДНОМ СВОЙСТВЕ РАСПРЕДЕЛЕНИЯ БРОУНОВСКОГО ДВИЖЕНИЯ СО СНОСОМ И ЕГО МАКСИМУМА}

Рассматривается задача максимизации математического ожидания функционала $P_{t} / \max _{0 \leqslant s \leqslant T} P_{s}$ на отрезке $[0, T]$, где $P_{t}=\exp \left\{\mu t+\sigma B_{t}\right\}-$ геометрическое броуновское движение. Показано, что в зависимости от сноса $\mu$ максимум достигается на одном из концов отрезка $[0, T]$.

Ключевые слова и фразы: броуновское движение, модель БлэкаШоулса, правило «Buy and Hold».

1. Введение. Рассмотрим модель Блэка-Шоулса, полагая, что относительные цены $P=\left(P_{t}\right)_{t \geqslant 0}$ подчиняются геометрическому броуновскому движению:

$$
d P_{t}=\left(\mu+\frac{\sigma^{2}}{2}\right) P_{t} d t+\sigma P_{t} d B_{t}, \quad P_{0}=1,
$$

на фильтрованном вероятностном пространстве $\left(\Omega, \mathscr{F},\left(\mathscr{F}_{t}\right)_{t \geqslant 0}, \mathbf{P}\right)$, где $B=\left(B_{t}\right)_{t \geqslant 0}$ стандартное броуновское движение по мере $\mathbf{P}$, а $\mathscr{F}_{t}=\sigma\left(B_{s}, s \leqslant t\right)$ - естественная фильтрация. Из формулы Ито следует, что

$$
P_{t}=\exp \left\{\mu t+\sigma B_{t}\right\} .
$$

В работе [1] рассматривается задача

$$
\sup _{\tau \in \mathfrak{M}^{T}} \mathbf{E}\left(\frac{P_{\tau}}{\max _{0 \leqslant s \leqslant T} P_{s}}\right),
$$

где $\mathfrak{M}^{T}$ - множество моментов остановки относительно фильтрации $\left(\mathscr{F}_{t}\right)_{t \geqslant 0}$, принимающих значения в $[0, T]$. В статье $[2]$ рассматривается аналогичная «детерминистическая» задача исследования на максимум на отрезке $[0, T]$ функции

$$
F(t)=\mathbf{E}\left(\frac{P_{t}}{\max _{0 \leqslant s \leqslant T} P_{s}}\right) .
$$

Исходя из графиков $F(t)$ при некоторых значениях параметров, в [2] делается предположение о том, что максимум достигается на одном из концов отрезка. Далее нами будет приведено аналитическое доказательство этого факта. В отличие от указанной статьи, для нахождения исследуемой функции $F(t)$ вместо потраекторного интегрирования используются теорема Гирсанова и принцип отражения.

* Московский государственный университет им. М.В.Ломоносова, механикоматематический факультет, Ленинские горы, 119991 Москва, ГСП-1, Россия; e-mail: almurav@gmail.com 